\title{
Measuring apparatus for determinate absorption of the thin-film coatings by the photothermal method
}

\author{
Galyna A. Petrovska, Igor V. Demkovych, Yaroslav V. Bobitski \\ Department of Photonics, Lviv Polytechnic National University \\ 12 Bandera Str., 79013 Lviv, Ukraine \\ Phone: 8-032-2582581, e-mail: galina_petrovska@mail.ru
}

The optical elements of high-power lasers and lasers systems must have the limiting low absorption so as heat that appears at the absorption produces the thermal deformations of the surfaces of the optical elements; change the spatial, energy and spectral parameters of emission and destruction of the optics.

The problems that are connected with production of the high-quality laser optics expect to the creation of the highly sensitive nondestructive testing methods of absorption in the optical elements, to low the absorption of the thin-film coatings.

In this paper the results development of noncontact nondestructive method for measuring low absorption in thin-film coatings are presented. This method is based on using photothermal effects.

If the researcher sample will be irradiate a powerful laser radiation, as a result of part of radiation will be absorbed, and the thermal fields appears there, the spatial-temporal distributing of which depends both on the parameters of laser radiation and descriptions of material of sample. The local increase the temperature of sample results to the different sort of the photostimulated changes in it and environment: thermodeformation of surface, infrared emission, thermal lens in sample and air, acoustic wave and others. In works [1 - 4] we are developed the photothermal methods of diagnostics of optical and thermophysical descriptions of materials after the experimentally registered thermal fields or deformation fields which appears at the irradiation the researcher sample by the powerful laser radiation beam with the known spatial-temporal distributing of radiation.

The value of absorbed power laser irradioation is determined by the value of thermal lens in the air near the surface of the researcher sample. The thermal lens as the result of the emission of the power laser irradiation with known spatial-temporal distribution of emission. The scheme of the thermal lens in air (a) and interferogram of lens that was registered by Michelson interferometer (b) (see Fig. 1).
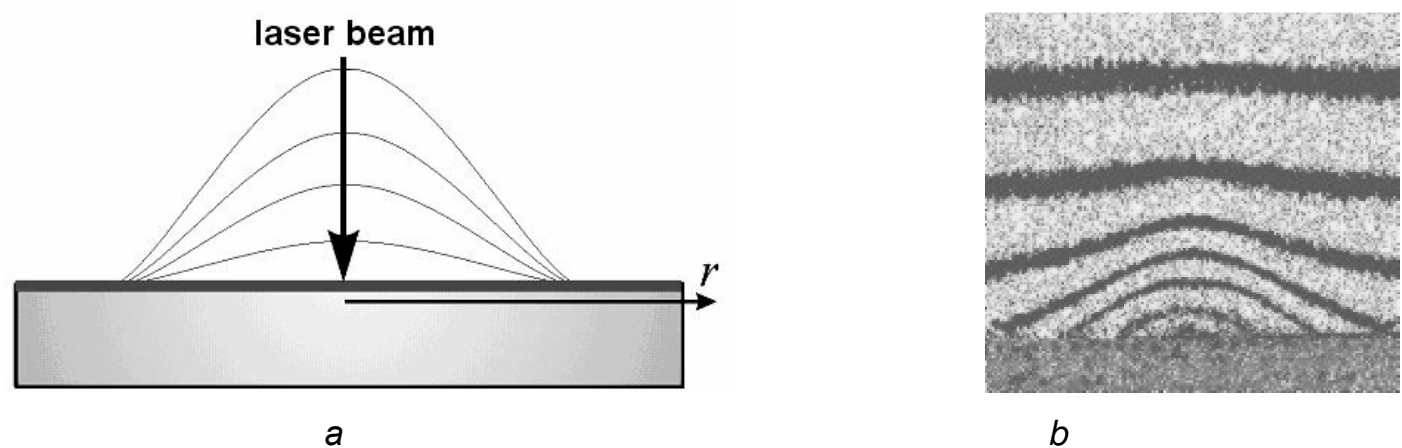

Fig. 1. The thermal lens in air:

$a$ - scheme representation; $b$ - interferogram of the thermal lens.

The scheme of the plant for the measurement absorption of the thin-film coatings is presented on Fig. 2. The registration of the thermal lens is used the two-channel scanning Fabry-Perot interferometer. The researcher sample 3 is emitted by the beam of the high-power laser. The energy is measured by photodetector 11. The single-frequency He-Ne-laser 2 is used as testing. The beam of testing laser is divided on two parallel beams by splitter 5 . These beams are on input of the scanning interferometer (mirrors 6 and 7) and one with it parallel pass to the surface of the research sample through the thermal lens (measuring channel II), the second - abroad of the thermal lens (reference channel I). 


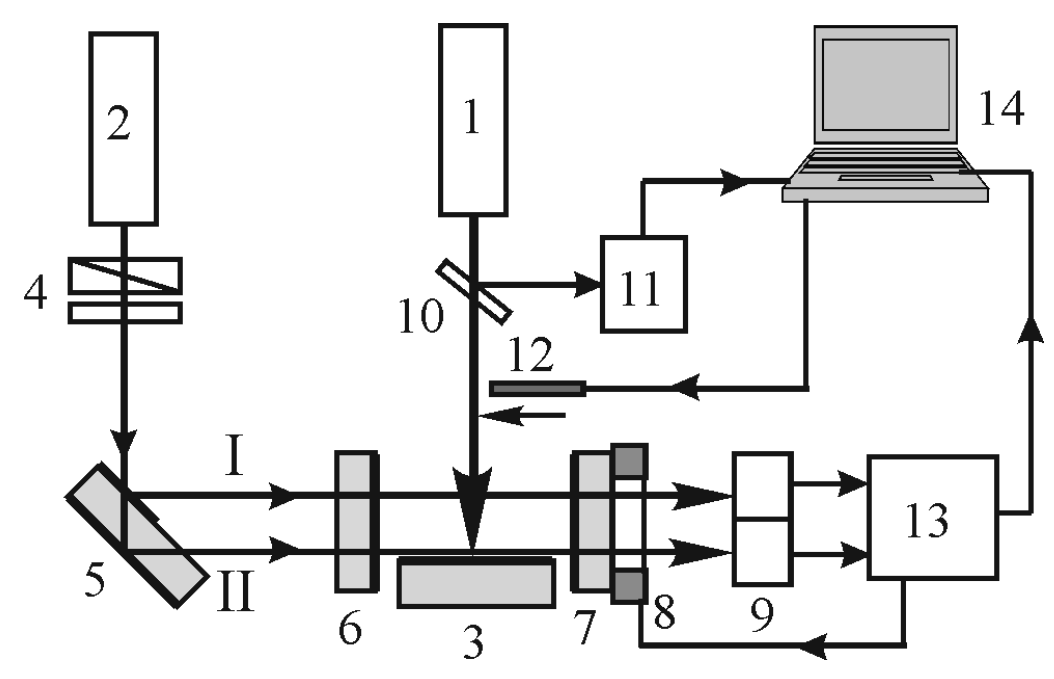

Fig. 2. The plant for the measurement absorption of the thin-film coatings: 1 - driven laser, 2 - testing laser, 3 - researcher sample, 4 - optical shutter, 5 - divider, 6, 7 - mirrors of interferometer of FabryPerot, 8 - piezoelectric, 9 - photodiode, 10 - divider, 11 - photodetector, 12 - shutter, 13 - phasemeter, $14-\mathrm{PK}, \mathrm{I}$ - reference channel of the interferometer, II - measuring channel

The changes of phase $\Delta \varphi_{e}(r)$, that appear in the measuring channel II at emission of the researcher sample 3 by power laser beam 1 are experimental registered by the scanning interferometer Fabry-Perot. The system registration of the shift of the interference bands for measurement of the phase changes is developed. The operating principle of this system is based on use of the temporal interval method $[5,6]$.

The signals from the both channels of the interferometer are registered by two-area photodiode 9 . These signals forms the two sequences of thin rectangular impulses in the phasemeter 13 . The phasemeter include microprocessor system that measuring different in phases between the reference and measuring channel of interferometer. The time diagrams that show work of the phasemeter are presented on Fig. 3.

The first measurement of different the phase between the two channels executes at closed shutter, the second - after opening shutter 12. The phase changes in the measuring channel of the interferometer that arisen as result this appearance in the channel of thermal lens are equal:

$$
\Delta \varphi_{e}(r)=\Delta \varphi_{2}-\Delta \varphi_{1}
$$

$\Delta \varphi_{1}, \Delta \varphi_{2}$ - the initial difference of the phases and the difference of phases after establishment of the thermal lens.

The initial difference of the phases across temporal intervals (Fig. 3 ) is determined as:

$$
\begin{gathered}
T=\frac{t_{2}+t_{3}}{2}-\frac{t_{1}}{2} ; \quad \Delta t=\frac{t_{4}+t_{5}}{2}-\frac{t_{1}}{2} \\
\Delta \varphi_{o}=\arccos \left(\frac{\Delta t}{T}\right)=\arccos \left(\frac{t_{2}+t_{3}-t_{1}}{t_{4}+t_{5}-t_{1}}\right) .
\end{gathered}
$$

The difference of the phases $\Delta \varphi_{2}$ is determined analogical to previously after establishment of the stationary thermal lens at the continuous emission of the researcher sample.

The principle of the determination of the unknown value absorption of the thin-film coatings consists following way: mathematical computer-simulated field of the temperatures and induced the phase 
changes in measuring channel of the interferometer with help mathematical optimization methods [7] aproxmated to the experimental registered phase changes.

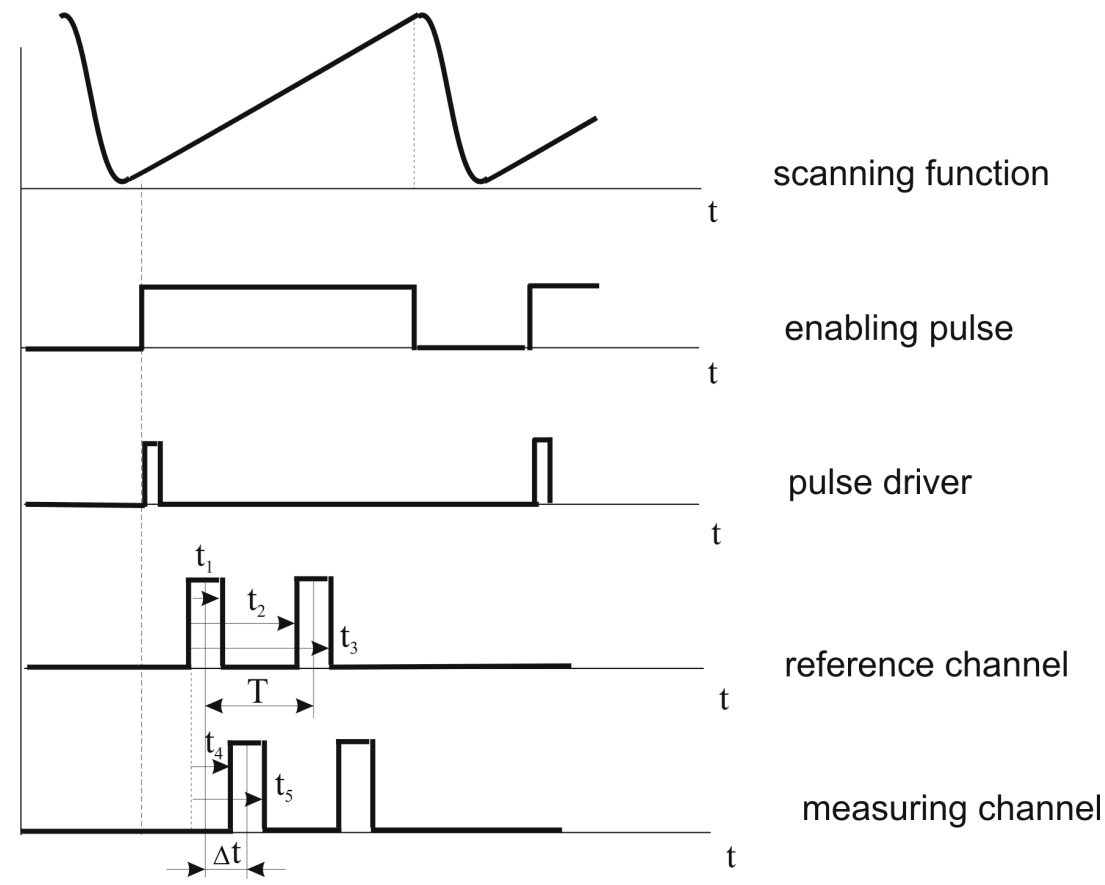

Fig.3. The time-diagrams that show work of the phasemeter

The minimum of the next functional corresponds to modal value of absorption:

$$
\Phi=\int_{0}^{l}\left[\Delta \varphi_{e}(r)-\Delta \varphi_{t}(r, \alpha)\right]^{2} d r
$$

where $\Delta \varphi_{e}(r)$ - experimental registered phase change, $\Delta \varphi_{t}(r, \alpha)$ - phase change that theoretically calculated for the certain value of the absorption $\alpha$ :

$$
\Delta \varphi_{t}(r, \alpha)=\frac{2 \pi l}{\lambda} \cdot \gamma \int_{0}^{l}\left[T_{2}(r, \alpha)-T_{1}(r)\right]^{2} d r,
$$

where $T_{1}(r)$ i $T_{2}(r, \alpha)$ - temperature distribution in air along coordinate $r$ in first time and in given after interaction with the power laser irradiation; $l$ - geometrical length of ray path in measuring arm of the interferometer; $\gamma$ - coefficient of thermal increment of the refractive index of air.

The calculation of temperature distribution on the surface of the researcher sample $T_{2}(r, \alpha)$ is provided by numerical methods using the mathematical model that was developed and described in works [1-4]. Software is developed based on this mathematical model, that allows designing the temperature fields which appears in the sample at operating on it of laser radiation with given spatialtemporal parameters.

Piezocorrector KP1-1 is used as a transformer for realization of scanning interferometer. Advantage is possibility of receipt of the large migration (within the limits of a few lengths of waves $\lambda$ ) without the use of high-voltage enregy source. The frequency of scanning is near $100 \mathrm{Hertz}$. The migration to the one length of wave $\lambda$ is arrived at the peak value of voltage about $10 \mathrm{~V}$. It is allows to use precision analog technique for measuring and easily to provide differential non-linearity less than $0.5 \%$.

The optimum function of scanning for piezocorrector of the scanning interferometer for expansion of range of measuring values and increase of accuracy of measuring is modeled. This optimum function is characterized the large linear region and small harmonic content [8]. 
The sensitivity of method in a large measure depends on accuracy of measuring of difference of phases between two channels of interferometer. The most exact measuring can be got, if signals from the output of interferometer have a regular and stable shape and large porosity. However, if as a testing laser is a multifrequency laser, than at the base of interferometer of Fabry-Perot near 5sm appears an external selection of longitudinal cavities resonance of measuring laser which causes in channels of interferometer of faulty impulses. The sensitiveness of phasemeter is $\lambda / 1000$ at the use of single-frequency testing laser with low noise level.

This method characterizes high the sensitivity and accuracy, absence the influence of the light scattering and not need the calibration. The experimental researches shown that when use the argon laser with power $5 \mathrm{~W}$ for emission and the single-frequency He-Ne laser as testing then the sensitivity of the measurement absorption of the thin-film coatings is $0,005 \%$ at the relative error not more than $10 \%$.

\section{References}

1. G.A. Petrovska, I.V. Demkovych, Y.V. Bobitski, et al. "The complex method for determination of the absorption of the optical materials", Ukrainskyj Fizychnyj Dzurnal, vol.46, №8, p.893, 2001.

2. G. A. Petrovska, I. V. Demkovych, Y. V. Bobitski. Photothermal method for measurement of thermal and physical parameters of construction materials Proc. $1^{\text {st }}$ International conference on advanced optoelectronics and lasers (CAOL'2003).- Alushta, Crimea South Coast (Ukraine).2003.-P.150-152.

3. G.A. Petrovska, I.V. Demkovych, Y.V. Bobitski "Photothermal method for determination of the absorption of the mirror coatings", Visnyk Lviv Polytechnic National University. Electronika, №532, p.170, 2005.

4. Petrovska H., Demkovych I., Bobitski Y. The measurement photothermal method of mirrors covering absorption coefficient// Proc. $7^{\text {th }}$ International Conference on Optical Technologies, Optical Sensors and Measuring Techniques OPTO 2006 - Nürnberg, Germany 2006, 147-152pp.

5. Pat. SSSR №1522865 Device for measuring of fractional part of interference band //Martynevych G.A. (G.A. Petrovska), Marusyn T.V., Tyschenko P.A. // - № 4417450, G 01 B 9/02.

6. Pat. SSSR №1468207 Digital phasometer//Martynevych G.A. (G.A. Petrovska), Marusyn T.V., Tyschenko P.A. // - № 4281969, G 01 R 25/08.

7. Terry E. Shoup, A practical guide to computer methods for engineers. - N. J.: Prentice-Hall, inc. Englewood Cliffs, 1979. - 238 c.

8. Tataryn Ya.V., Petrovska G.A. Optimization of function scanning of piezocorrector of the scanning interferometer// Proc. $11^{\text {th }}$ Conference with problem electronics - Lviv, Ukraine 2008, p. 38 